\title{
Potential pathogen transmission on medical student anatomy laboratory clothing
}

\author{
Chandan J. Kabadi ${ }^{1}$, Carroll R. Smith III', Fernando Gomez ${ }^{2 *}$ \\ ${ }^{1}$ American University of the Caribbean School of Medicine, Cupecoy, St. Maarten \\ ${ }^{2}$ Department of Pathology, American University of the Caribbean School of Medicine, Cupecoy, St. Maarten \\ *Corresponding author: Fernando Gomez MD; fgomez@aucmed.edu
}

\begin{abstract}
Introduction: Despite great advances in the fields of medicine and sanitation, nosocomial infections remain a very common and serious issue. Many of these problems can be avoided by simple hand washing; however, pathogenic microbes can spread through other modes too. In our study, we aim to determine if the setting of an open cadaver laboratory was conducive to the transmission of pathogens such as Staphylococcus aureus, Streptococcus pyogenes, and Enterococcus faecalis.

Methods: For this investigation, 67 volunteer medical students had their laboratory coats swabbed and sampled during their time in anatomy laboratory class. Each coat was sampled prior to cadaver contact and at the end of their time in the laboratory, which coincided with the exploration of the gastrointestinal tract.

Results: We found that pathogens were present on the laboratory coats of the students. An increase in each of the three microbes for which we tested was detected at the end of the anatomy laboratory course on the garments of the participants. There were six more student laboratory coats with $\mathrm{S}$. aureus in the post-dissection swabbing and there were three more student laboratory coats with S. pyogenes in the post-dissection swabbing than originally documented. E. faecalis was found on four student laboratory coats in the post-dissection swabbing compared to none pre-dissection.

Discussion: From these results, we conclude that stronger infection control measures are warranted to prevent the occurrence of unnecessary disease transmission in this setting. Our study provides data that support further investigation of potential pathogen transmission by student laboratory clothing and supports the use of universal infection control procedures to provide safer environments for medical students and their contacts, including laundering protocols for coats.
\end{abstract}

Keywords: pathogen transmission; infectious precautions; white coat; medical students; contamination; cadaver.

\section{INTRODUCTION}

N osocomial infections within hospitals and clinics remain an important topic of discussion. The role of hand washing in limiting disease transmission in the healthcare setting, as well as in the community, is well understood. ${ }^{1-3}$ However, the role of clothing worn by medical students in disease transmission is not as well understood.

Infections that result from bacteria do not have to be foreign to the human body itself. Multiple sites in the body may contain Staphylococcus aureus, and it is mostly frequently carried in the anterior nares of the nose. Previous studies have estimated that $20 \%$ of the human population is a long-term carrier of S. aureus. ${ }^{4}$ Streptococcus pyogenes, a ubiquitous microorganism that frequently colonizes throats of asymptomatic people, was found with carriage rates of 15-20\% in infants and $10 \%$ in adult smokers. ${ }^{5}$ As a normal colonizer of the gut, Enterococcus faecalis is also commonly found. One study performed on the feces of adult patients showed the presence of $E$. faecalis in $48.2 \%$ of adult outpatients and $80 \%$ of adult inpatients. ${ }^{6}$

While some species of bacteria are found in the normal flora, those same bacteria may gain resistance to antibiotics and become a danger to healthcare workers and patients. It has been demonstrated that methicillin-resistant S. aureus (MRSA) and vancomycinresistant Enterococci (VRE) can be found on the clothes of healthcare workers, particularly on sleeves, waist areas, and neckties. ${ }^{3,7}$ Consequently, guidelines have been established by the Centers for Disease Control (CDC) in the United States and the National Health Service (NHS) in the United Kingdom for the proper handling of clothing worn by healthcare workers, particularly visibly soiled clothes. ${ }^{1-3,8}$ Notably, the NHS has instituted a 'bare below the elbows' policy that prohibits long sleeves for clinical healthcare workers. ${ }^{8}$ Interestingly, the literature is silent on the occurrence of microorganism transmission via clothing 
worn by medical students studying anatomy in cadaver laboratories.

Executed properly, the process of cadaveric preservation using common embalming agents such as formalin, ethanol, and phenols is believed to eliminate the presence and growth of bacterial microorganisms, although some uncertainty exists regarding the post embalming infectious potential of hepatitis viruses, human immunodeficiency virus (HIV), and prions. ${ }^{9-11}$ Thus, it is believed that potential bacterial transmission via properly prepared cadaveric tissues is highly unlikely.

It is now common practice in medical education to assign a group of students to each cadaver, necessitating a close working environment for students. Physical encounters such as sleeves of laboratory coats brushing together are inevitable in such a close working environment. It is common in some institutions for medical students to wear or carry their scrubs and/or laboratory coats to and from the anatomy laboratory. Microorganisms acquired on scrubs and laboratory coats could potentially be transmitted to others if non-laundered garments are transported or worn outside of the confined environment of the anatomy laboratory.

It is currently unknown whether microorganisms can be transmitted in this manner. If such transmission proves to be frequent, the transmission of potentially pathogenic microorganisms is of concern to medical schools around the world. Furthermore, investigation of laboratory coats and scrubs for easily spread nosocomial pathogens, such as S. aureus, S. pyogenes, and $E$. faecalis, could warrant further studies into the detrimental effects caused by their transmission. It may also warrant changes in anatomy laboratory procedures, which may include changing the transport and washing procedure of laboratory garments and restricting the ability to take the garments outside of the anatomy laboratory to minimize patient and community exposure to potential pathogens. These changes could also directly affect procedures in hospitals and clinics.

\section{METHODS}

Medical students who were enrolled in the anatomy course at the American University of the Caribbean School of Medicine in St. Maarten were solicited to voluntarily participate in this experiment prior to beginning the anatomy laboratory segment of the course. In order to guarantee confidentiality, participants were assigned a random number and linked to that number in a database accessible only to the investigators. Students were instructed to wear their laboratory coats at all times inside the anatomy laboratory. Outside of the laboratory, students were not directed on how to manage their laboratory coats and were advised to maintain their normal cleaning and laundering routines. Students were not informed when the samplings would be conducted in order to prevent any changes to their routine that could potentially alter results.

Typical student management of laboratory coats involves bringing the laboratory coats from their place of residence to the laboratory and then leaving with the coats. Laboratory coats are not kept in the laboratory, and while regular cleaning of the laboratory coats was encouraged by the faculty, there were no specific requirements and cleaning regimens of individual students were not monitored. Thus, individual management of laboratory coats by students was expected to vary.

Culture samples were obtained from the sleeves and front of the laboratory coats on the participant's dominant side using sterile saline-moistened swabs. A culture sample was collected before the start of the first anatomy laboratory session prior to any engagement between the students and the cadavers. Another sample was collected from all participating medical student laboratory coats toward the end of the anatomy course, specifically after the gastrointestinal tract was explored by the students, which was 50 days after the initial sampling.

Each sample was first inoculated onto blood agar plates directly from the swab. After incubation for 48 hours at $37^{\circ} \mathrm{C}$, distinctive colonies were then separately cultured on fresh blood agar plates for 24 hours at $37^{\circ} \mathrm{C}$. Colony morphology and hemolytic patterns were observed for each isolate. Isolates were then subcultured on mannitol salt agar (MSA; 24 hours at $37^{\circ} \mathrm{C}$ ), Streptococcus-selective agar (SSA; 24 hours at $\left.37^{\circ} \mathrm{C}\right)$, and bile esculin agar (BEA; 24 hours at $37^{\circ} \mathrm{C}$ ). Colony and gram stain characteristics of the organisms, along with subsequent catalase and coagulase testing, were then utilized to identify the different species of bacteria present from each swabbing using their respective bacterial properties. ${ }^{12}$

The results were then analyzed by chi-squared analysis and a standard t-test. The chi-squared analysis was done to compare the data as a nominal type defined by whether or not bacteria were present before and after dissection. A one tailed standard ttest was also done to see if the mean of bacterial presence on the laboratory coats was significant between the control and post-dissection data to justify further investigation with a larger sample size. 


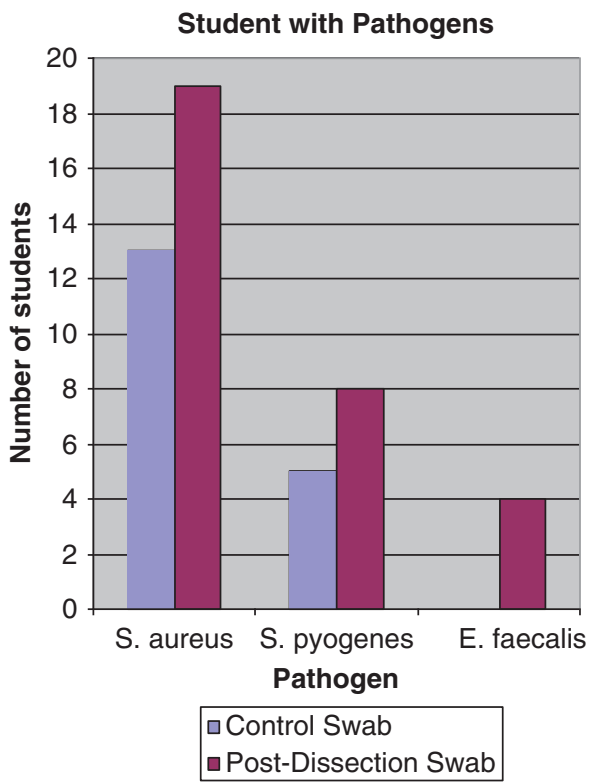

Figure 1. Pathogenic presence on laboratory coats based on organism type and number of students with microorganism presence.

\section{RESULTS}

Initial sampling, which was done before students had entered the anatomy laboratory, showed that $S$. aureus was found on the laboratory coats of 13 of 67 students (19.4\%), S. pyogenes was found on coats of 5 of 67 students (7.46\%), and E. faecalis was not found on any the coats of the 67 students (0\%) (Fig. 1). In the laboratory coat sampling done after the cadaver was dissected and the gastrointestinal tract was exposed, laboratory coats of 19 of 67 students were found to have S. aureus ( $28.4 \%), 8$ were found to have S. pyogenes $11.9 \%)$, and 4 were found to have E. faecalis (5.97\%).

There were 6 more student laboratory coats with S. aureus in the post-dissection swabbing than in the first sampling, which is a $46 \%$ relative increase. There were 3 more student laboratory coats with S. pyogenes in the post-dissection swabbing compared to the initial sampling, which is a $60 \%$ relative increase.

\section{DISCUSSION}

The investigation began with the control swabbing, which would represent the normal conditions for which a laboratory coat was maintained. The alternative hypothesis was that the student laboratory coats would have a greater incidence of each bacterial type after exposure to cadavers than before any dissection occurred. The null hypothesis was that there
Table 1. Analysis of control swabbing $(n=67)$.

\begin{tabular}{lccc}
\hline & Absolute count & Mean & Standard deviation \\
\hline S. aureus & 13 & 0.194 & 0.159 \\
S. pyogenes & 5 & 0.075 & 0.070 \\
E. faecalis & 0 & 0.000 & 0.000 \\
\hline
\end{tabular}

would be no difference before or after exposure to cadavers. This experiment was designed as a one-tailed test, since the chance of disinfection as a result of cadaver exploration was not plausible.

For all laboratory coat swabbings, a value of 0 was assigned if the particular bacteria did not grow on the specific media and a value of 1 was given if the bacteria were present. Data from the control and the postdissection swabbings are shown in Tables 1 and 2, respectively. To check the significance of the data, a Chi-square analysis of the data and a two sample $t$-test of the means were carried out.

For the Chi-square analysis, the post-dissection swabbing was considered the observed value, and the control was the expected value. Table 3 describes the Chi-square analysis carried out for $S$. aureus that showed a $P$-value of 0.064 , which was not statistically significant. Table 4 for $S$. pyogenes showed a $P$-value of 0.163 , which was also not statistically significant. Table 5 showed the analysis for $E$. faecalis, which had to be done in two parts. Because the control value for the amount of $E$. faecalis found was 0 , the Chi-square analysis could not be completed without a division by 0 error (as noted in Table 5). To assess the significance of the observed $E$. faecalis data, the expected bacteria found value was changed to 1 in order to calculate the limit of the $P$-value as the expected value approached 0 . For the expected value of 1 , the $P$-value was $<0.003$, which was statistically significant. However, the Chisquare analysis is highly dependent on the sample size. While each swabbing group had a sample size of 67 , the results suggest that the number of students would need to be increased in order to have a more definitive conclusion; a greater number of student participants while maintaining the same proportion of laboratory

Table 2. Analysis of post-dessection swabbing $(n=67)$.

\begin{tabular}{lccc}
\hline & Absolute count & Mean & Standard deviation \\
\hline S. aureus & 19 & 0.284 & 0.206 \\
S. pyogenes & 8 & 0.119 & 0.107 \\
E. faecalis & 4 & 0.060 & 0.057 \\
\hline
\end{tabular}


Table 3. Chi-square for Staphylococcus aureus on laboratory coats.

\begin{tabular}{|c|c|c|c|c|c|}
\hline & Observed (o) & Expected (e) & Deviation $(d)$ & Deviation $^{2}\left(d^{2}\right)$ & $\left(d^{2}\right) / e$ \\
\hline No bacteria & 48 & 54 & -6 & 36 & 0.667 \\
\hline Bacteria found & 19 & 13 & 6 & 36 & 2.769 \\
\hline \multirow[t]{2}{*}{$n=67, \mathrm{df}=1$} & & & & $X^{2}$ & 3.436 \\
\hline & & & & $P$ & 0.064 \\
\hline
\end{tabular}

coats with and without bacteria would result in statistical significance for all bacteria tested.

In order to justify further investigation into this subject, we wanted to make sure that the difference in proportions between the pre- and post-dissection swabbing was significant. Therefore, a one-tailed two sample $t$-test of the means was conducted. Table 6 demonstrates the analysis of the $t$-test for the three tested bacteria. The results show that the mean of the post-dissection swabbing was higher than predissection swabbing, with statistical significance for all three bacteria.

The laboratory coats tested in this study were purchased by students approximately 2 weeks before the start of the anatomy laboratory class; therefore, the coats were purchased 2 weeks before any solicitation was done for participation in the experiment. During the 2 weeks, the laboratory coats would have been exposed to the environment outside of the laboratory for enough time that it was assumed the control would be representative of any outside contamination. Thus, the presence of $S$. aureus and S. pyogenes in the initial samples was not surprising as they are commonly found in the normal upper respiratory tract flora. E. faecalis, which is part of the normal intestinal flora, was not expected to be on laboratory coats from environmental sources, which was confirmed by the control swabbing.

In the post-dissection swabbing, more students were found to have S. aureus, S. pyogenes, and E. faecalis on their laboratory coats. The time between the first day sample and the post-dissection sample was 50 days. During this time, the laboratory coats were exposed to dissection of the anatomy cadaver, including the most recent exploration of the gastrointestinal tract. The presence of $E$. faecalis on the laboratory coats in the post-dissection samples was not expected based on the assumption that students practiced acceptable sanitary and laundering measures in their day-to-day lives. The absence of $E$. faecalis in the control and its presence in the post-dissection swabbing implies that while the sanitary practices were acceptable, laundering was a concern.

In conclusion, while it is hard to attribute a specific source to the transmission of S. aureus, S. pyogenes, and $E$. faecalis to laboratory clothing from this study, the results do support the need for further investigation as there was an increase in bacterial acquisition on the laboratory coats. In addition, the findings indicate that laboratory garments worn in the anatomy laboratory setting were not sterile after exposure to the cadaver and thus harbor potentially pathogenic microorganisms. Given the current findings, proper timely laundering of laboratory clothing is recommended. The garments used in the anatomy laboratory should not be used for other activities, such as patient-clinical encounters, clinical interviews, diagnostic skills courses, or other formal activities requiring the use of laboratory coats, without first assuring that the garments have been properly disinfected.

Improvements could be made on the collection of data. As seen in the analysis, the Chi-square showed that only the presence of $E$. faecalis was statistically significant. A factor limiting the significance of the S. aureus and S. pygoenes data was the sample size. The analysis of the means through the $t$-test showed that the change in proportions for the appearance of bacteria on laboratory coats pre- and post-dissection

Table 4. Chi-square for S. pyogenes on laboratory coats.

\begin{tabular}{|c|c|c|c|c|c|}
\hline & Observed (o) & Expected (e) & Deviation $(d)$ & Deviation $^{2}\left(d^{2}\right)$ & $\left(d^{2}\right) / e$ \\
\hline No bacteria & 59 & 62 & -3 & 9 & 0.145 \\
\hline Bacteria found & 8 & 5 & 3 & 9 & 1.800 \\
\hline \multirow[t]{2}{*}{$n=67, \mathrm{df}=1$} & & & & $x^{2}$ & 1.945 \\
\hline & & & & $P$ & 0.163 \\
\hline
\end{tabular}


Table 5. Chi-square for E. faecalis on laboratory coats.

\begin{tabular}{|c|c|c|c|c|c|}
\hline \multicolumn{6}{|c|}{ A - Using actual values } \\
\hline & Observed (o) & Expected (e) & Deviation $(d)$ & Deviation $^{2}\left(d^{2}\right)$ & $\left(d^{2}\right) / e$ \\
\hline No bacteria & 63 & 67 & -4 & 16 & 0.239 \\
\hline Bacteria found & 4 & 0 & 4 & 16 & ERROR \\
\hline \multicolumn{6}{|c|}{ B - Assuming expected bacteria found value is 1} \\
\hline & Observed (o) & Expected (e) & Deviation $(d)$ & Deviation $^{2}\left(d^{2}\right)$ & $\left(d^{2}\right) / e$ \\
\hline No bacteria & 63 & 66 & -3 & 9 & 0.136 \\
\hline Bacteria found & 4 & 1 & 3 & 9 & 9.000 \\
\hline \multirow{2}{*}{$n=67, \mathrm{df}=1$} & & & & $X^{2}$ & 9.136 \\
\hline & & & & $P$ & $<0.003$ \\
\hline
\end{tabular}

Table 6. Two-sample $t$-test for means of swabbings ${ }^{\circ}$.

\begin{tabular}{lcccc}
\hline & Mean & Standard deviation & $\mathrm{t}$ & $P^{*}$ \\
\hline $\begin{array}{l}\text { S. aureus } \\
\text { Post-dissection }\end{array}$ & 0.284 & 0.206 & 2.831 & 0.0025 \\
$\begin{array}{l}\text { Control } \\
\text { S. pyogenes }\end{array}$ & 0.194 & 0.159 & & \\
$\begin{array}{l}\text { Post-dissection } \\
\text { Control }\end{array}$ & 0.119 & 0.107 & 2.817 & 0.0028 \\
$\begin{array}{l}\text { E. faecalis } \\
\text { Post-dissection }\end{array}$ & 0.075 & 0.070 & & \\
Control & 0.000 & 0.057 & & \\
\hline
\end{tabular}

Values obtained from Tables 1 and 2.

*One-sided $P$-value calculation.

does justify further research and investigation into this matter. It would be our recommendation that the sample size be increased in order to obtain more conclusive results. While the means were analyzed using the $t$-test to justify further studies, the true significance of this study would be justified with significant Chi-square results due to the binomial nature of the data.

There were possible confounding variables that also warrant further investigation. The laboratory coats were not documented to which cadaver they were working with. There were 10 cadavers in the anatomy laboratory, and it is unknown if the positive laboratory coats were all in the same vicinity or worked with the same cadaver. In addition, no tests were performed on the cadavers themselves for the presence of bacteria.

Since the laboratory coats left the laboratory on a daily basis, the method of cleaning could not be standardized. While the control was designed to minimize any environmental contribution to the post- dissection swabbing, it is not possible to fully eliminate the environment as a possible contaminant given the current laundering protocol. This still leaves the environment outside the anatomy laboratory as a possible source of bacterial acquisition.

While there were variables that need to be better controlled, the experiment did show that there was a significant increase in the bacteria between pre- and post-dissection swabbings. It is the belief of the experimenters that the bacteria were transmitted from the anatomy cadavers. Whether due to the cadavers, environmental exposures, or improper laundering, the increased presence of bacteria on the laboratory coat does assert the need for specific laboratory coat cleaning protocols and warrants further investigation to prove the source of bacterial acquisition on the laboratory coats.

Acknowledgements: We would like to acknowledge Dr. Lancelot Nash, Dr. Kathleen Shupe and Ms. Christine Headland for their assistance in this project; Jason Crawford for research done to provide support for this research project; Pallavi Nadendla, Theodoros Pantazis, Fatoumata Sakho, Haroon Shaukat and David Stawski for their assistance in helping analyze, as well as collect, samples for this experiment.

Conflict of interest and funding: Funding for this project was provided by the American University of the Caribbean with no conflicts of interest. This study has been approved by the American University of the Caribbean Institutional Review Board. This project was carried out in accordance with the Declaration of Helsinki, including there being no potential harm to any participant, the anonymity of participants being guaranteed, and the informed consent of all participants was obtained prior to any research conducted. 


\section{REFERENCES}

1. CDC (2002). Guideline for hand hygiene in health care settings: recommendations of the Healthcare Infection Control Practices Advisory Committee and the HICPAC/SHEA/ APIC/IDSA Hand Hygiene Task Force. Vol. 51, No. RR-16. Atlanta, GA: Centers for Disease Control and Prevention. 2. Siegel J, Rhinehard $E$, Jackson $M$, Chiarello $L$, the HICPAC. Management of multidrug-resistant organisms in healthcare settings. Atlanta, GA: Centers for Disease Control; 2006. http://dx.doi.org/10.1016/j.ajic.2007.10.006.

3. Siegel J, Rhinehart E, Jackson M, Chiarello $L$, the HICPAC. Guideline for isolation precautions: preventing transmission of infectious agents in healthcare settings. Atlanta, GA: Centers for Disease Control; 2007. http://dx.doi.org/10.1016/ j.ajic.2007.10.007.

4. Kluytmans J, van Belkum A, Verbrugh H. 1997. Nasal carriage of Staphylococcus aureus: epidemiology, underlying mechanisms, and associated risks. Clin Microbiol Rev 10(3):505-20.

5. Sevillano D, Aguilar L, Alou L, Giménez MJ, González N, Torrico $M$, et al. 2008. $\beta$-Lactam effects on mixed cultures of common respiratory isolates as an approach to treatment effects on nasopharyngeal bacterial population dynamics. PloS One 3(12):e3846. http://dx.doi.org/10.1371/

journal.pone.0003846.

6. Noble C. 1978. Carriage of group D streptococci in the human bowel. J Clin Pathol 31:1182-6. http://

dx.doi.org/10.1136/jcp.31.12.1182.
7. Nurkin S, Urban, C, Mangini, E, Mariano, N. Is the clinician's necktie a potential fomite for hospital acquired infections [abstract]. 104th General Meeting of the American Society for Microbiology. 2004; 23-27.

8. National Health Service Department of Health. Uniforms and workwear: an evidence base for developing local policy. National Health Service Department of Health; 2007. Available from: http://webarchive.nationalarchives.gov. uk/+/www.dh.gov.uk/en/publicationsandstatistics/ publications/publicationspolicyandguidance/dh_078433 9. O'Sullivan E, Mitchell B. 1993. An improved composition for embalming fluid to preserve cadavers for anatomy teaching in the UK. J Anat 182(Pt. 2):295-7.

10. Whitehead M, Savoia M. 2008. Evaluation of methods to reduce formaldehyde levels of cadavers in the dissection laboratory. Clin Anat 21(1):75-81. http://

dx.doi.org/10.1002/ca.20567.

11. Demiryurek D, Bayramoglu A, Ustacelebi S. 2002. Infective agents in fixed human cadavers: a brief review and suggested guidelines. Anat Rec 269:194-7. http://dx.doi.org/ 10.1002/ ar.10143.

12. Watson, R. General \& medical microbiology: summary of biochemical tests. University of Wyoming; 2013. Available from: http://www.uwyo.edu/molb2210_lab/info/ biochemical_tests.htm 